Lvov, etal., "Design and mechanical properties of 3D-printed auxetic honeycomb structure", Materials Today Communications, v. 24, (2020) https://doi.org/10.1016/j.mtcomm.2020.101173

\title{
DESIGN AND MECHANICAL PROPERTIES OF 3D-PRINTED AUXETIC HONEYCOMB STRUCTURE
}

\author{
V.A. Lvov' ${ }^{1}$, F.S. Senatov ${ }^{1}$, A.M. Korsunsky ${ }^{2,3}$, A.I. Salimon ${ }^{1,3}$ \\ ${ }^{1}$ National University of Science and Technology "MISIS", 119049, Leninsky pr. 4, Moscow, Russia \\ ${ }^{2}$ Department of Engineering Science, University of Oxford, Parks Road, Oxford OX1 3PJ, UK \\ ${ }^{3}$ HSM laboratory, Center for Energy Science and Technology, Skolkovo Institute of Science and \\ Technology (Skoltech), Moscow 121205, Russia
}

Correspondence to: Ivov.va@misis.ru, 119049, Moscow, Leninsky pr. 4, +7-495-638-44-13

\section{Abstract}

The combination of theoretical calculations, computer simulations, and experimental evaluation of Poisson's ratio was carried out for re-entrant honeycomb auxetic structure. Optimal honeycomb cell parameters were determined for 3D-printed samples made from thermoplastic polyurethane (TPU). The low-cycle compression tests of 3D-printed re-entrant honeycomb auxetic samples showed that the structure based on auxetic hexagonal cell can withstand almost 1.75 times more very low cycle fatigue cycles than the similar non-auxetic structure. Neither failure nor layer delamination in 3D structures were detected in the auxetic sample after 500 compression cycles. 3D-printed auxetic structures offer a promising candidate for applications in medicine and sports.

Keywords: Auxetics; Cycle loading; Deformation and fracture; Simulation and modeling

\section{Introduction}

Auxetic structures with negative values of Poisson's ratio $(v)$ attract attention due to their unusual deformation response, energy absorption properties, and durability under long term loading. In three-dimensional auxetic isotropic structures, the value of $v$ lies between -1 and $+1 / 2$. For two-dimensional 
Lvov, etal., "Design and mechanical properties of 3D-printed auxetic honeycomb structure", Materials Today Communications, v. 24, (2020) https://doi.org/10.1016/j.mtcomm.2020.101173

structures this value lies in the range -1 to 1 . For anisotropic materials and structures $v$ is not delimited, and can range from minus infinity to unity $[1,2]$.

Various variants of auxetic metamaterials and structures [3] have been proposed and fabricated in an attempt to target a wide range of potential applications, from microwave absorption [4] to medical prostheses [5]. Many suggested applications are motivated by the specific deformation mechanisms of auxetic structure. The use of auxetic structures/materials as viscoelastic dampers was considered in [6], in which a biphasic auxetic composite is reported to show a loss tangent exceeding the lower Voigt limit and close to the upper boundary of the Hashin bound.

Another prospective application of auxetic structures is for elements of body protection from impact loading for ensuring safety in construction industry, sport, and battle. Damping elements in protective helmets against shock have incorporated auxetic foams [7], ensuring that when the material is compressed in one direction, it also shrinks in others, thus resisting fragmentation and reducing the likelihood or severity of injuries.

A variety of structures and metamaterial structures showing the auxetic effect have been proposed. The most common classes are two-dimensional and three-dimensional frames [4,9], chiral structures [10], rotating rigid or semi-rigid structures, and carbon fiber laminate materials [11]. The class of auxetics derived from a honeycomb cell $[12,13]$ has been one of the most explored due to the simplicity of fabricating three-dimensional structures of complex shape by 3D-printing.

The majority of literature reports on auxetics are limited to the theoretical modelling of strain behavior of auxetic structures with additional ribs, and by the computer simulations of experiments using Finite Element Analysis (FEA). To the best of the authors' knowledge, no reports on cyclic tests for 3Dprinted two-dimensional or three-dimensional auxetic structures and metamaterials appear to have been published up to date. However, FEA computation of 3D (or 4D) printed structures has been reported recently in the work of Oladapo et al [19] in the study of the effect of ultrasound on the microstructure of samples.

Also, reports have been published on the effect of negative Poisson's ratio on mechanical properties. For example, Jiang et al [17] investigated experimentally and numerically the compressive strength of polymer nets for 3D printing with simple cylindrical cubic (CSC), simple cubic quadrangular 
Lvov, etal., "Design and mechanical properties of 3D-printed auxetic honeycomb structure", Materials Today Communications, v. 24, (2020) https://doi.org/10.1016/j.mtcomm.2020.101173

(FSC) and mixed simple cubic (MSC) unit cells before and after metallization of the surface. The aim of the work was to determine the dependence of the compressive strength of polymer nets on the structure, and the increase in strength due to the electrodeposition of metal films.

The current study presents theoretical calculation, computer simulations, and experimental measurements of Poisson's ratio $v$ in a re-entrant honeycomb auxetic structure. Very low cycle compression tests of the 3D-printed re-entrant honeycomb auxetic structure were performed for the first time.

\section{Materials and Methods}

\subsection{Design of auxetic and non-auxetic structures}

For the purposes of geometric and numerical analysis, the unit cells with honeycomb auxetic (shown in solid lines in Fig.1A) andnon-auxetic structures (inversion of A shown by the dashed line) can be described using the following parameters:

$h$, the length of the horizontal strut

I, the length of the inclined strut

$\theta$, the inclination angle;

$t$, the cross-sectional diameter (thickness) of the horizontal and inclined struts (Fig. $1 \mathrm{~A})$.
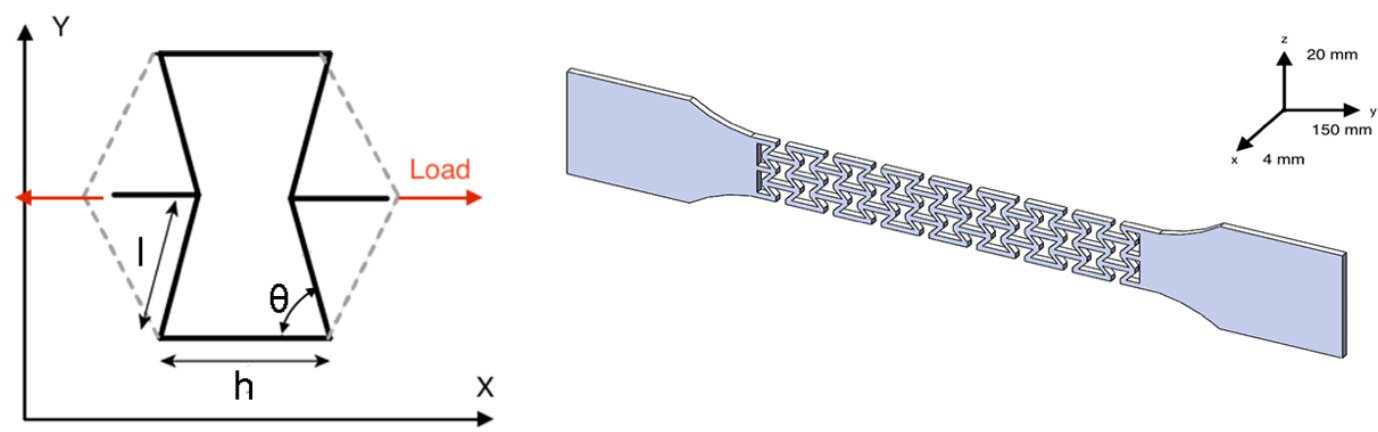

A

B

Figure 1. (A) Theoretical geometry model of the unit cell (A) re-entrant auxetic (solid line) and nonauxetic (dashed line) cell and (B) single layer plane model for experimental measurements. 
Lvov, etal., "Design and mechanical properties of 3D-printed auxetic honeycomb structure", Materials Today Communications, v. 24, (2020) https://doi.org/10.1016/j.mtcomm.2020.101173

For small strains, Poisson's ratio of the honeycomb auxetic structure can be calculated as the ratio of the transverse compressive strain to the longitudinal extension strain:

$$
v=-\frac{\varepsilon_{x}}{\varepsilon_{y}}=-\frac{\sin ^{2} \theta}{(\alpha-\cos \theta) \cos \theta}
$$

Here $\alpha=h / l$ and $\theta$ are geometric parameters related to the unit cell geometry shown in Fig.1.

\section{$I \cos \theta$}

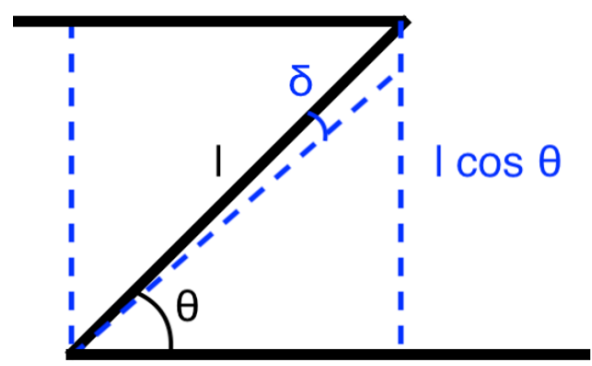

$\mathrm{h}$

Figure 2. Illustration for the derivation of eq. (1) .

The derivation of equation (1) is made in accordance with the illustration in Fig. 2 by calculating the longitudinal $\left(\varepsilon_{x}\right)$ and transverse $\left(\varepsilon_{y}\right)$ strains as:

$$
\begin{gathered}
\varepsilon_{x}=-\frac{l \delta \sin \theta}{(h-l \cos \theta)} \\
\varepsilon_{y}=\frac{l \delta \cos \theta}{l \sin \theta} \\
v=-\frac{\varepsilon_{x}}{\varepsilon_{y}}=-\frac{l \delta \sin \theta}{(h-l \cos \theta)} \frac{l \sin \theta}{l \delta \cos \theta}=-\frac{l \sin ^{2} \theta}{(h-l \cos \theta) \cos \theta}=-\frac{\sin ^{2} \theta}{(\alpha-\cos \theta) \cos \theta} .
\end{gathered}
$$

\subsection{Computer Simulation}

Alongside the trigonometric evaluation of strains in the unit cell geometry, computer modelling was conducted using Autodesk Fusion 3602018 software (Autodesk, Inc. Santa Monica, CA, USA) for a periodically repeated unit cell. Tensile and compressive tests were simulated in accordance with ISO 5271/ASTM D638 for the one-layer plane model of $150 \times 20 \times 4 \mathrm{~mm}$ dimensions (Fig.1B) to calculate Poisson's 
Lvov, etal., "Design and mechanical properties of 3D-printed auxetic honeycomb structure", Materials Today Communications, v. 24, (2020) https://doi.org/10.1016/j.mtcomm.2020.101173

ratio v. Finite Element mesh included 5203 nodes and 2195 elements. Convergence tolerance of $5 \%$ was used. The same model was also used as input for 3D-printing of samples for experimental measurements of Poisson's ratio $v$ in tension.

\subsection{Fabrication of test specimens and mechanical testing}

Thermoplastic polyurethane (molecular weight of $100000 \mathrm{~g} / \mathrm{mol}$, REC 3D, Russia) filament with diameter of $1.75 \mathrm{~mm}$ was used for FDM 3D-printing by means of 3D-printer BiZon Prusa i3 Steel PRO (3DiY, Russia). Feed rate of $15 \mathrm{~mm} / \mathrm{sec}$, nozzle temperature of $225^{\circ} \mathrm{C}$, and print bed temperature of $60^{\circ} \mathrm{C}$ were maintained during 3D printing. Samples with inclination angle $\vartheta$ varying over in the range 30 to 70 degrees and $\alpha=2$ were fabricated by 3D-printing.
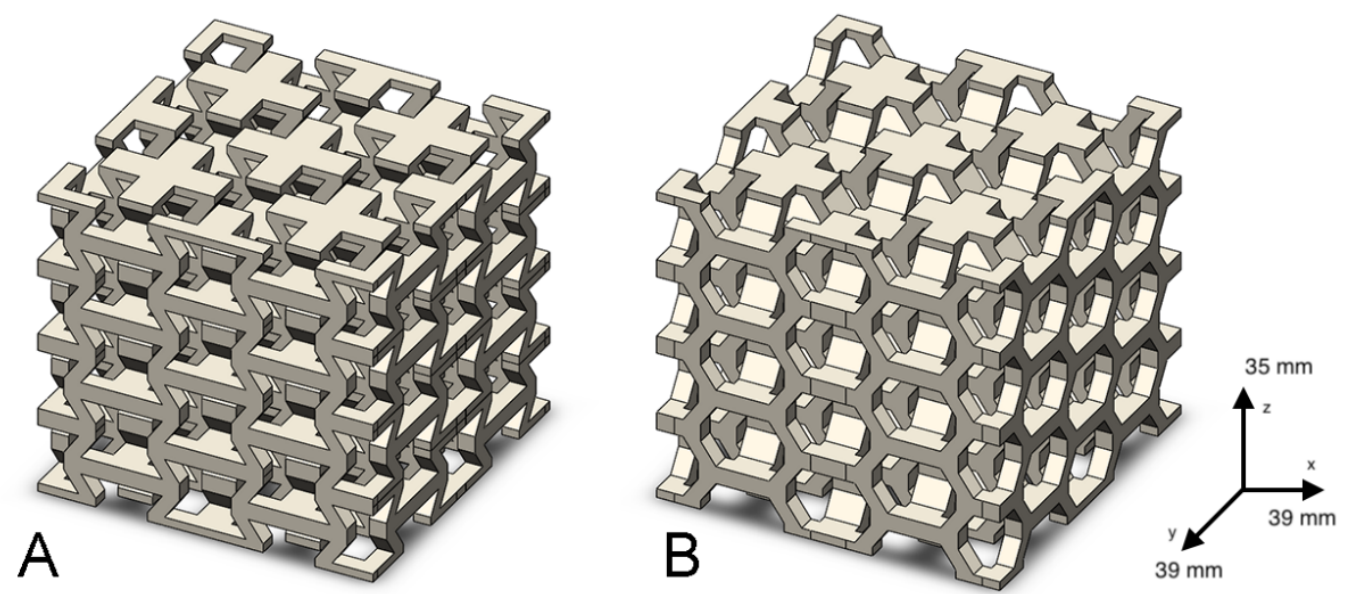

Fig 3. Models for 3D-printed auxetic (A) and non-auxetic structure (B).

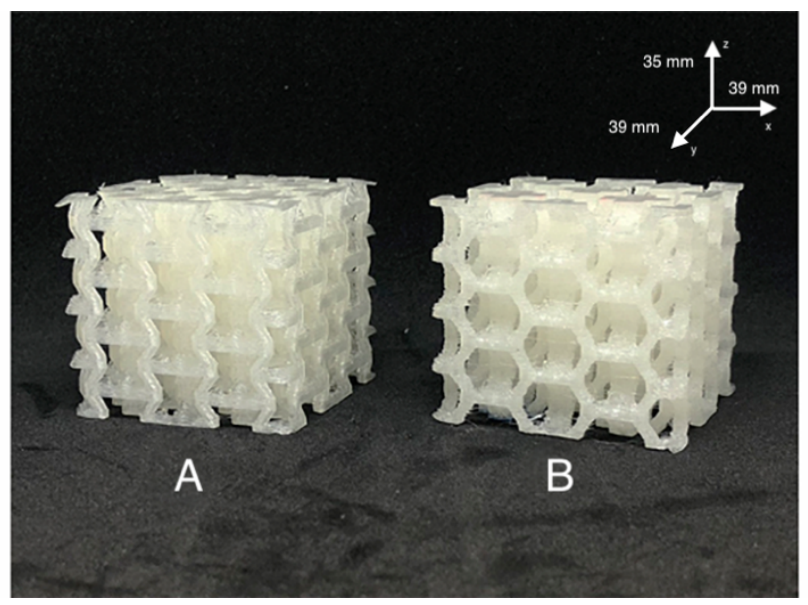

Fig. 4. Samples of 3D-printed auxetic $(A)$ and non-auxetic structure (B) 
Lvov, etal., "Design and mechanical properties of 3D-printed auxetic honeycomb structure", Materials Today Communications, v. 24, (2020) https://doi.org/10.1016/j.mtcomm.2020.101173

Poisson's ratio measurements were conducted using a universal testing machine Zwick/Roell Z020 (Zwick GmbH \& Co. KG, Germany). Additional gauges for monitoring longitudinal and transverse displacements were utilized to evaluate $v$ for 3D-printed 150×20×4 mm samples.

Cyclic compression tests were performed for the samples of rectangular cross-section with the dimensions of $39 \times 39 \times 35 \mathrm{~mm}$ (Fig 2,3). Very low cycle fatigue tests (500 cycles) were performed at the crosshead speed of $10 \mathrm{~mm} / \mathrm{min}$ under controlled displacement range of $5 \mathrm{~mm}$ ("hard" cycle mode).

\subsection{Microscopy}

Samples were sectioned after cyclic compression testing, and microscopic images of the nodes of auxetic and non-auxetic structure were collected using a scanning electron microscope (Hitachi TM 1000).

\section{Results and Discussions}

\subsection{Calculation of Poisson's ratio}

Using equation (1), the dependence between $v$ and $\theta$ was obtained for the range of inclination angle between 10 and 80 degrees (Fig. 5). For $\alpha=2$, negative Poisson's ratio decreases monotonically with increasing $\theta$, with the minimum module value of $v$ attained at $\theta=10^{\circ}$. It should be noted that the character of theoretical dependence changes depending om parameter $\alpha$. In agreement with the theoretical predictions, $v$ varies in a range from 0 to -0.5 in computer simulation results. The minimum value of $v$ for this set of results was found at a smaller value of the inclination angle $\left(\theta=60^{\circ}\right)$.

The values of $v$ obtained from computer simulations and experiment shown in Fig.5 are quite close to the estimates from the theoretical analysis for the smaller values of inclination angles. Theoretical calculations in accordance with (1), however, ignore the fact that the struts connecting the nodes experience deformation that may fail into plastic regime, making $v$ dependent on the value of sample global strain. It can be concluded that $v$ tends to be a dynamic quantity applicable for a certain range of inclination angles $\theta$. Finally, based on the results presented, angle $\theta$ of $55^{\circ}$ was chosen as the optimal angle to fabricate samples having 3D auxetic and non-auxetic structures. 
Lvov, etal., "Design and mechanical properties of 3D-printed auxetic honeycomb structure", Materials Today Communications, v. 24, (2020) https://doi.org/10.1016/j.mtcomm.2020.101173

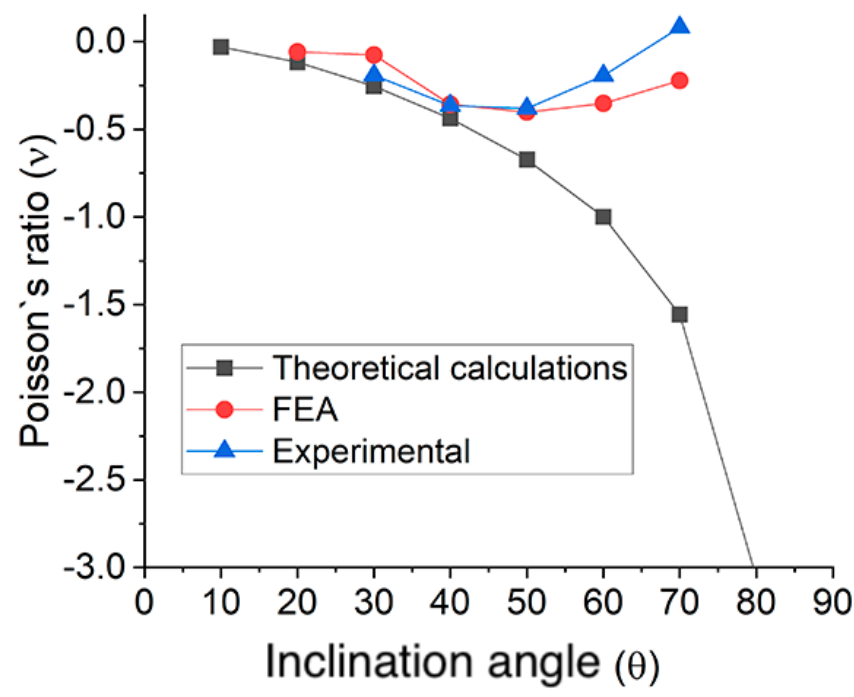

Fig. 5 The dependence of the Poisson's ratio ( $v)$ on the inclination angle ( $)$

\subsection{Cyclic tests}

The results of cyclic tests (Fig. 6 A, B) show that auxetic (A) and non-auxetic (B) structures exhibit similar mechanical properties under primary loading up to strain of $14 \%$ when the nominal stress value reaches $12 \mathrm{MPa}$. However, these structures behave differently under cyclic loading. The area of the hysteresis loop of the non-auxetic structure is several times bigger than that of auxetic counterpart due to the fact that the non-auxetic sample did not restore its shape completely upon unloading. It can be assumed that auxetic structures distribute the load throughout the frame volume in more even manner.

Under cyclic compression the non-auxetic sample acquires a "barrel-like" appearance, while the sample with auxetic structure eventually collapses under compressive load due to the elastic buckling and loss of stability (Fig. 6 C, D). 
Lvov, etal., "Design and mechanical properties of 3D-printed auxetic honeycomb structure", Materials Today Communications, v. 24, (2020) https://doi.org/10.1016/j.mtcomm.2020.101173
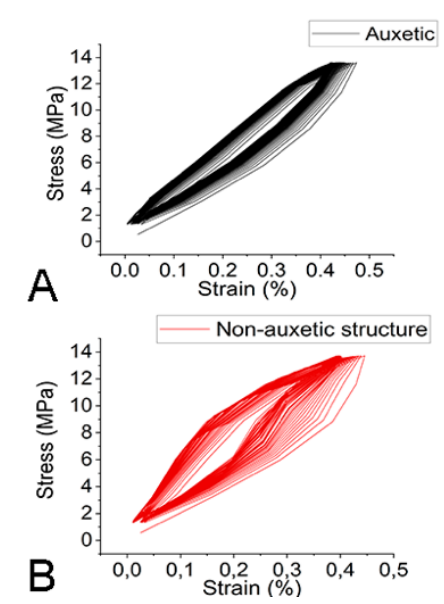
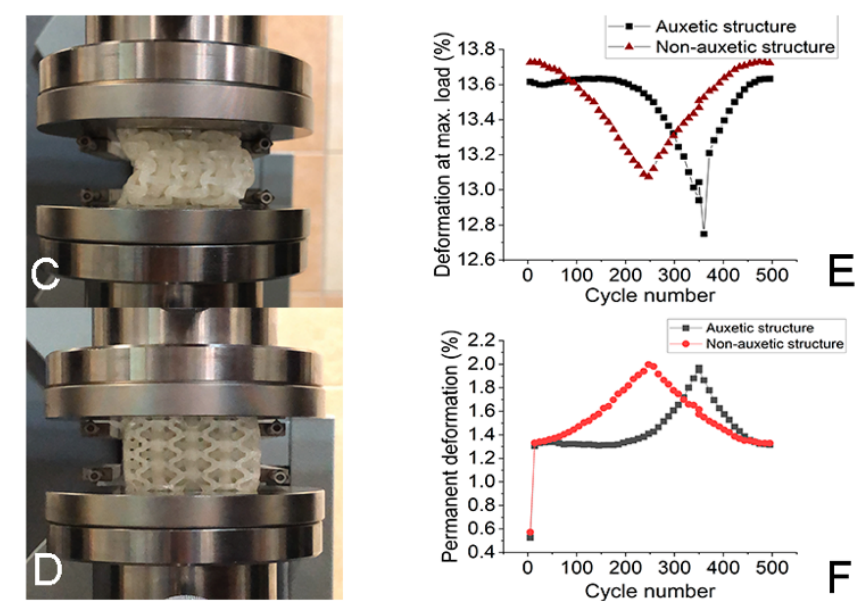

Fig. 6. Stress-strain dependence under cyclic compression for (A) auxetic, and (B) non-auxetic structures,

The appearance of $(C)$ auxetic and $(D)$ non-auxetic structures after compression. Residual deformation and maximum deformation vs the number of cycles for (E) auxetic and (F) non-auxetic structure

It is worth to note that the deformation of the auxetic structure decreased by $0.8 \%$ after 350 cycles and at the same time there was a $0.6 \%$ peak in the residual deformation (Fig. 6 E). The non-auxetic structure shows a similar drop of deformation value (from 13.7 to $13.1 \%$ ), while the residual deformation increased from 1.4 to $2.0 \%$ after 200 cycles (Fig. 6 F).

In this case, the amount of absorbed mechanical energy of the auxetic and non-auxetic structures is close in value and amounts to $8 \mathrm{~J}$ after 500 cycles (Fig. 7).

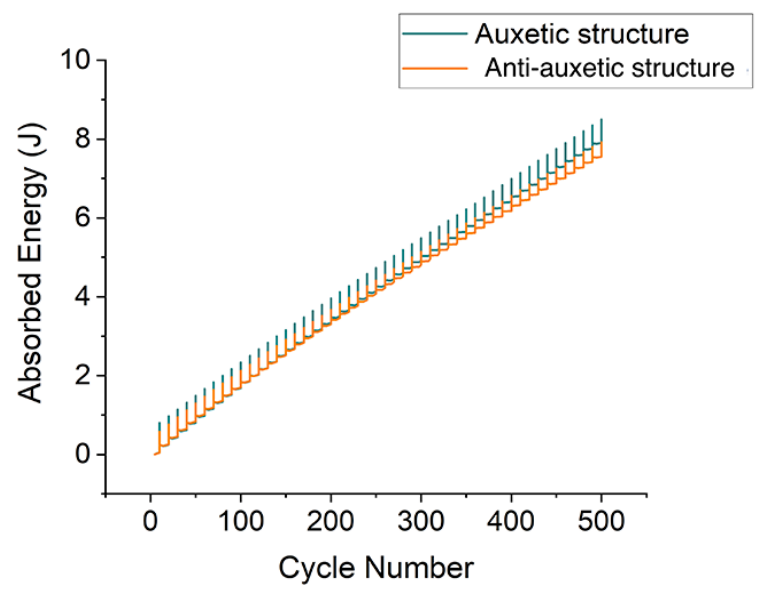

Fig. 7. The dependence of absorbed mechanical energy (J) the number of cycles for auxetic and non- 
Lvov, etal., "Design and mechanical properties of 3D-printed auxetic honeycomb structure", Materials Today Communications, v. 24, (2020) https://doi.org/10.1016/j.mtcomm.2020.101173

SEM images do not reveal any reliably detectable damage or signs of deterioration at the nodes in both geometric structures (Fig. 8).
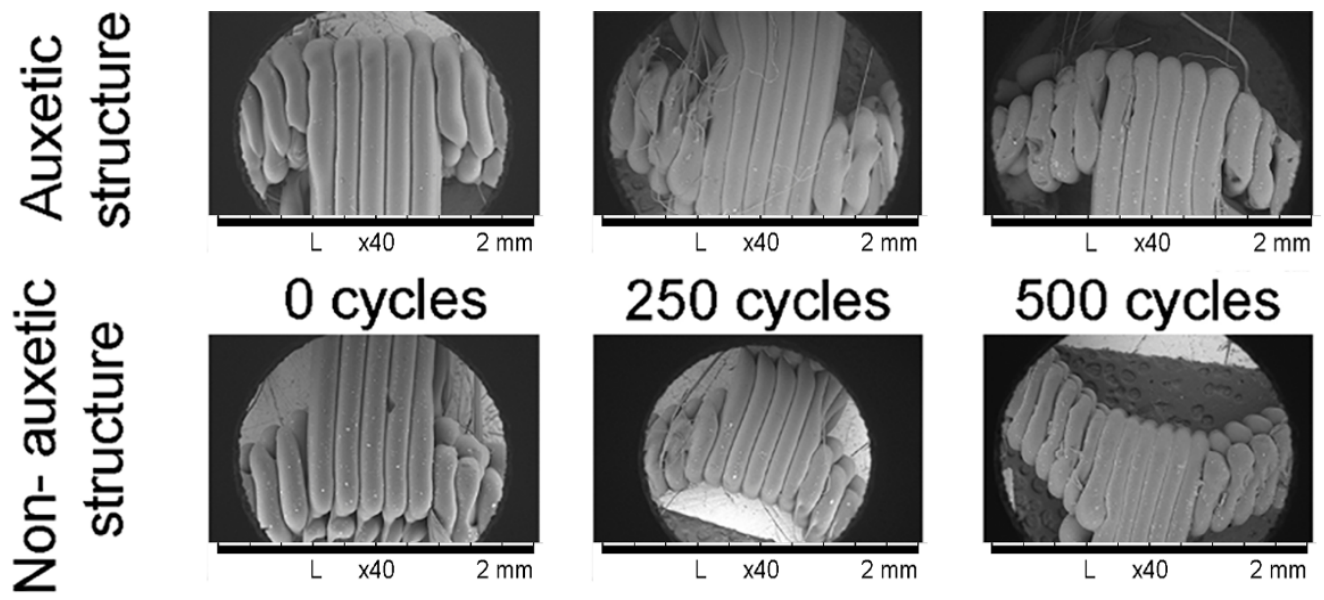

Fig. 8. Scanning electron microscopy images of samples at key points of cyclic tests.

The dissipation of accumulated work of deformation is believed to unfold by means of heat release, damage evolution, and ultimate fatigue failure. The latter is likely to happen during longer term cycling till final rupture. One can expect both deformation hardening and softening effects to take place at the nodes where highest strains are concentrated. Logical further steps to investigate these structures would involve operando experiments, such as thermography, high resolution video recording with DIC analysis, and small angle X-ray scattering analysis at synchrotron facilities. The investigation of viscoelastic phenomena at the nodes of auxetic structures will provide key insights into the possible smart mechanical applications of auxetic metamaterials.

\section{Conclusions}

According to the literature sources, auxetics can be considered as structures capable of absorbing and dissipating mechanical, sound, and thermal energy. The key parameter for such structures is Poisson's ratio and its minimal value that could be considered optimal for mechanical applications. However, there are no general analytical formulae for describing it. In spite of this, the re-entrant auxetics represent an 
Lvov, etal., "Design and mechanical properties of 3D-printed auxetic honeycomb structure", Materials Today Communications, v. 24, (2020) https://doi.org/10.1016/j.mtcomm.2020.101173

example that can be described from the basic geometric point of view as a preliminary step for sophisticated analysis.

Computer simulation facilitates the search for optimal geometric parameters for a re-entrant auxetic cell and finding the minimal value of Poisson's ratio. It was experimentally shown that under compression cycling auxetics dissipate less work of deformation since they are likely distributing the load throughout the volume more evenly and not concentrating strain at the nodes. Studied auxetic structures potentially can be used as "dampers" for better mechanical energy dissipation due to the ability to absorb smaller amount of energy compared to non-auxetic structures.

\section{References}

[1]. Cobras L., Brun M. Auxetic two-dimensional lattice with Poisson's Ratio arbitrarily close to -1 ", Proceedings of the Royal Society a Mathematical, Physical and Engineering Sciences. 470 (2014)

[2]. Cabras L., Brun M. Effective properties of a new auxetic triangular lattice: an analytical approach, Frattura ed Integrità Strutturale, 2014, pp. 9-18.

[3]. Smith C. W., Grima J. N. A novel mechanism for generating auxetic behaviour in reticulated foams: missing rib foam model. Acta. Mater, 2000, pp. 4349-4356.

[4] Chen, Lakes Micromechanical Analysis of Dynamic Behavior of Conventional and Negative Poisson's Ratio Foams, Journal of Engineering Materials and Technology, 1996, pp. 285-288.

[5]. Scarpa F., Giacomin J. Mechanical performance of auxetic polyurethane foam for antivibration glove applications, Cell. Polym, 2005, pp. 253-268.

[7]. Daniel I. M. \& Ishai O. Engineering mechanics of composite materials, Oxford University Press, 1994

[8]. Grima J. N., Gatt R. On the potential of connected stars as auxetic systems, Molecular Simulation, 2005, pp. 925-935.

[9]. Grima J.N., Gatt R. On the properties of auxetic meta-tetrachiral structures, Phys. Status Solidi B, 2008, pp. 511-520.

[10]. Spadoni, A., Ruzzene, M. Global and local linear buckling behavior of a chiral cellular structure, Physica status solidi, 2005, pp. 695-709. 
Lvov, etal., "Design and mechanical properties of 3D-printed auxetic honeycomb structure", Materials Today Communications, v. 24, (2020) https://doi.org/10.1016/j.mtcomm.2020.101173

[11]. Hine P.J., Duckett R.A. Negative Poisson's ratios in angle-ply laminates, Journal of Materials Science Letters, 1997, pp. 541-544.

[12]. Liu Y., Hu, H. (2010). A Review on Auxetic Structures and Polymeric Materials, Scientific Research and Essays, 2010, pp. 1052-1063.

[13]. Yang W., Li Z.-M. Review on auxetic materials, Journal of Material Science, 2004.

[14]. Gibson L.J., Ashby M.F. The mechanics of two-dimensional cellular materials, Proc. R. Soc. Land, pp. 25-42.

[15]. Huang J., Zhang Q. In-plane elasticity of a novel auxetic honeycomb design, Composite Structures, 2017, pp. 72-82.

[16]. Xin-Tao Wang, Xiao-Wen Li Interlocking assembled 3D auxetic cellular structures, 2016.

[17]. Pan Jiang, Zhongying Ji et al. High Compressive strength metallic architectures prepared via polyelectrolyte-brush assisted metal deposition on 3D printed lattices, Nano-Structures \& Nano-Objects, 2018, pp. 420-427.

[19] Bankole I. Oladapo, Edwin A. Oshin, Aderinsola M. Olawumi. Nanostructural computation of 4D printing carboxymethylcellulose (CMC) composite, Nano-Structures \& Nano-Objects, 2020, 21.

[20] Joseph P.V. et al. Short sisal fiber reinforced polypropylene composites: the role of interface modification on ultimate properties, Composite Interfaces, 2012.

[21] Bejoy Francis et al. Cure kinetics and morphology of blends of epoxy resin with poly (ether ether ketone) containing pendant tertiary butyl groups, Polymer, 2003, pp. 3687-3699.

[22] Kishor Kumar Sadasivuni et al. Dielectric properties of modified graphene oxide filled polyurethane nanocomposites and its correlation with rheology, Composites Science and Technology, 2014, pp. 18-25. 